\title{
Range-based Trilateration Using Multipurpose Cost Function Optimization with Lagrangian Multipliers
}

\author{
Ali Khalajmehrabadi, David Akopian, and Daniel Pack \\ Department of Electrical and Computer Engineering, University of Texas at San Antonio \\ One UTSA Circle, San Antonio, Texas 78249-0669, USA \\ Emails:\{ali.khalajmehrabadi, david.akopian, daniel.pack\}@utsa.edu
}

\begin{abstract}
In this paper, a novel trilateration positioning technique is proposed that jointly addresses the conventional rangebased trilateration localization and measurements outliers detection and processing. The proposed scheme contains three components: (a) detecting outliers, (b) minimizing outliers impact; (c) minimizing conventional noise impact. The method is based on a linear regression model in which the noise and outliers vector effect is considered simultaneously. The cost function includes an $\ell_{1}$-norm minimization component to detect outliers. Once detected, the contaminated ranges are either removed from measurements or corrected. The proposed scheme has been simulated and compared with the recently proposed Linearized LS (L-LS), range-based LS (R-LS), and Squared-Range Least Squares (SR-LS) approach. The results shows that the proposed approach is able to detect $97 \%$ of single introduced outliers and leads to less position error variance compared to the state-ofthe-art approaches.
\end{abstract}

\section{INTRODUCTION}

In recent years, location-based services (LBSs) and technologies evolved and enabled mobile applications from transportation to social media [1]. Proven positioning systems such as Global Positioning System (GPS) [2] and WLAN-based indoor localization [3]-[5] are commonly used in consumer, industry and military applications. Technology concepts vary and include trilateration techniques as in GPS [2], [6], [7], radio-fingerprint maps for WLAN-based systems [8], triangulation methods that explore the direction of arrival (DOA) estimation in terrestrial networks [9]-[11], etc.

Trilateration techniques exploit beacons at known locations and range measurements to find user locations. The positioning is performed using systems of equations connecting obtained beacon-to-user range measurements with geometric distances that include unknown user coordinates. Conventionally, the number of equations corresponds to the number of beacons, but other measurements can supplement these equations for more accurate estimations.

Trilateration methods are commonly subdivided in two categories: 1) closed form solutions [12] and 2) numerical methods [13], [14]. As the range measurements are typically noisy and there might be more beacons than unknowns, least squares (LS) solutions of overdetermined system of equations are often used to exploit redundancy and obtain more accurate results. Linear LS methods are used for both closed-form and iterative techniques. In general, a maximum-likelihood (ML) estimator is considered as an asymptotically optimal solution [15], but its hard to reach. The LS solutions coincide with the ML one for Gaussian deviations of range measurements, and asymptotically achieve the so-called Cramér-Rao lower bound (CRLB) that is introduced for such estimators [16], [17]. CRLB provides a lower bound on the variance achievable by any unbiased location estimator and is useful as a guideline [18]. Similar ideas are explored for collaborative localization and non-line-of-sight (NLOS) ranging bias models [19].

While LS optimization is a common approach of solving trilateration equations, the cost function of LS optimization is nonconvex as includes unknowns in nonlinear Euclidian distance functions, and hence, convergence to a global optimum is not guaranteed. Several recent studies proposed relaxed or alternative cost functions, such as squared range LS (SRLS) which allows for finding global optimum solutions [19] and [20]. These solutions guarantee global optimum of the cost function [21], but their performance is inferior to LS techniques when exact LS solution can be found [20].

In real environments range measurements are not necessarily Gaussian and LS solutions degrade in accuracy. It is also common to have outliers deviations in range-measurements. While previous studies have extensively investigated the filtering of outliers, they typically explore LS techniques to detect them.

This paper provides a novel trilateration method for agents in two dimensional (2D) space. The locations of these agents are to be determined through the range measurements obtained from anchor points with known locations. Rather than using the complicated nonlinear method, the trilateration system of equations is linearized. The location of agents are defined through an optimization technique that jointly estimate the pose of the agent, minimizes the noise, and detects the potential outliers in range measurements.

In this paper, the typical trilateration localization formulation is discussed in Section II. The proposed joint localization and outliers detection scheme is introduced in Section III as an extension to the conventional approach. Section IV illustrates the simulation performance of our method followed by conclusions in Section V.

\section{TRILATERATION LOCAlization CONCEPTS AND FORMULATION}

Consider a single agent with coordinates $(x, y)$ in $\mathcal{R}^{2}$ and range measurements $r_{i}, i=1, \ldots, N$, obtained from a set of 
anchor points $\mathcal{A}=\left\{A_{1}, \ldots, A_{N}\right\}$ through some mounted sensors that measure the mutual distances. The relation between the actual geometric distance and the range measurement can be expressed as

$$
\begin{aligned}
r_{i}=d_{i}+\epsilon_{i} & =\sqrt{\left(x_{i}-x\right)^{2}+\left(y_{i}-y\right)^{2}}+\epsilon_{i} \\
i & =1, \ldots, N
\end{aligned}
$$

where $d_{i}$ is the actual Euclidean distance between the robot and the anchors and $\epsilon_{i}$ is the corresponding noise component. The location of the robot $\hat{\mathbf{p}}=(\hat{x}, \hat{y})$ should be estimated through the following minimization problem

$$
\hat{\mathbf{p}}=\underset{\mathbf{p}}{\operatorname{argmin}} \sum_{i=1}^{N}\left(r_{i}-\left\|\mathbf{p}_{i}-\mathbf{p}\right\|\right)^{2}
$$

where $\mathbf{p}_{i}$ is the position of the $i$-th anchor and $\mathbf{p}$ is the robot location. The minimization problem (2) is known as the range-based least square (R-LS) problem and since it is non-convex, it cannot be solved trivially with the conventional methods. Some recent approaches have proposed approximate reformulation of (2). The above problem can be re-cast as a constrained quadratic optimization problem [22]. This equivalent reformulation is solved through semi-definite relaxation (SDR) approximation. However, if the rank conditions are far from unity, the solution degrades drastically. This has been shown with an example in [20].

Another approach for solving (2) is through the so called squared-range based least square (SR-LS) reformulation as

$$
\hat{\mathbf{p}}=\underset{\mathbf{p}}{\operatorname{argmin}} \sum_{i=1}^{N}\left(r_{i}^{2}-\left\|\mathbf{p}-\mathbf{p}_{i}\right\|^{2}\right)^{2}
$$

Although SR-LS, like the R-LS, is non-convex, a global solution can be achieved when it is transformed into a constrained least squares problem which belongs to generalized trust region sub-problem (GTRS) [20].

A more conventional approach of solving (2) is to linearize the problem near its solution and solve a linear system of equations for finding the solution. If this process is performed repetitively, the method is called iterative least square (ILS) solution which needs a good initial estimate.

\section{Proposed Multi-Purpose Localization Scheme}

The discussed reformulations are generally approximations that reach to the actual position if certain conditions are satisfied. However, in our approach, we use a reformulation of (2) that leads to a set of linear equations. To this end, we need to define another variable other than the robot coordinates. Squaring both sides of (2) yields

$$
r_{i}^{2}=R^{2}-2 x x_{i}-2 y y_{i}+\left(x_{i}^{2}+y_{i}^{2}\right) .
$$

Rearranging the sides of the above equation

$$
\begin{aligned}
x x_{i}+y y_{i}-0.5 R^{2} & =\frac{1}{2}\left(x_{i}^{2}+y_{i}^{2}-r_{i}^{2}\right) \\
i & =1, \ldots, N
\end{aligned}
$$

where $R=\sqrt{x^{2}+y^{2}}$ is the new variable depending on the robot coordinates. The equivalent set of linear equations of (2) can be written as an overdetermined system of equations as [23]:

$$
\mathbf{A x}=\mathbf{b}
$$

where

$$
\begin{array}{r}
\mathbf{A}=\left(\begin{array}{ccc}
x_{1} & y_{1} & -0.5 \\
\vdots & \vdots & \vdots \\
x_{N} & y_{N} & -0.5
\end{array}\right), \mathbf{x}=\left(\begin{array}{c}
x \\
y \\
R^{2}
\end{array}\right) \\
\mathbf{b}=\frac{1}{2}\left(\begin{array}{c}
x_{1}^{2}+y_{1}^{2}-r_{1}^{2} \\
\vdots \\
x_{N}^{2}+y_{N}^{2}-r_{N}^{2}
\end{array}\right)
\end{array}
$$

which is called linearized least squares (LLS) problem and can be solved using the following conventional quadratic minimization algorithm

$$
\begin{aligned}
\hat{\mathbf{x}} & =\underset{\mathbf{x}}{\operatorname{argmin}}(\mathbf{A x}-\mathbf{b})^{T}(\mathbf{A x}-\mathbf{b}) \\
& =\left(\mathbf{A}^{T} \mathbf{A}\right)^{-1} \mathbf{A}^{T} \mathbf{b} .
\end{aligned}
$$

This solution minimizes the effect of range errors on position estimates due to the noise. However, if the noise effect become considerable, the agent's position estimate degrades. The localization approach may experience the unavailability or inordinate pair-wise range measurements. These inordinate range measurements are called outliers. Since the agent is moving, outliers may occur due to obstacle blockage, loss of anchors, system software, malfunctions, and jamming. In such cases, range measurements are not trustable. If range measurements contain outliers, penalizing the residuals between the actual and geometric range measurements does not guarantee outlier rejection and positioning suffers dramatically from large localization errors. One should note that an autonomous system is not able to recognize the range measurements that contain the outliers.

We augment the trilateration localization approach of (8) so that the position of the agent is jointly estimated with the outliers. To this end, the model (1) is augmented so that it includes the outliers

$$
r_{i}=d_{i}+\epsilon_{i}+\kappa_{i} \quad i=1, \ldots, N
$$

where $\kappa_{i}$ is the component that accounts for the outlier. The vector equivalent of (9) for linearized LS yields

$$
\mathbf{b}=\mathbf{A x}+\kappa+\mathbf{n}
$$

where $\boldsymbol{\kappa}=\left[\kappa_{1}, \ldots, \kappa_{N}\right]^{T}$ is a vector indicating the outliers and is sparse since a few number of range measurements contain outliers. Hence, $\kappa$ can be jointly estimated with the agent's location

$$
(\hat{\mathbf{x}}, \hat{\boldsymbol{\kappa}})=\underset{\mathbf{x}, \boldsymbol{\kappa}}{\operatorname{argmin}}\left[\|\mathbf{b}-\mathbf{A x}-\boldsymbol{\kappa}\|_{2}+\lambda\|\boldsymbol{\kappa}\|_{1}\right]
$$

where the $\ell_{1}$-norm recovers a sparse vector for the outliers and $\lambda$ is a Lagrangian multiplier. This optimization formulation minimizes the difference between the coefficient matrix 


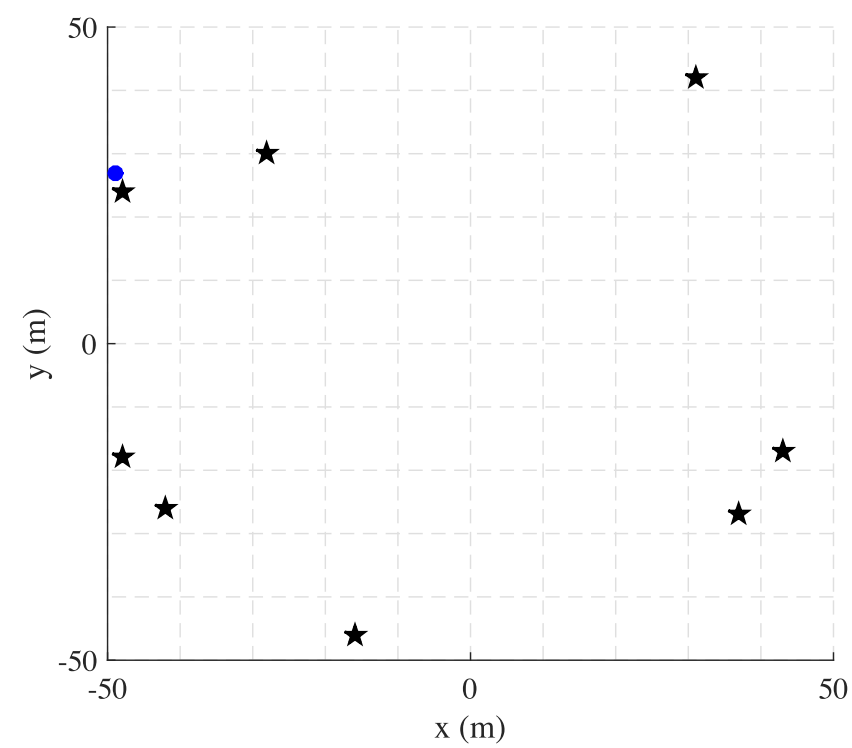

Fig. 1. A worst-case anchors and agent geometry. The black stars and the blue circle show the anchor locations and agent location, respectively.

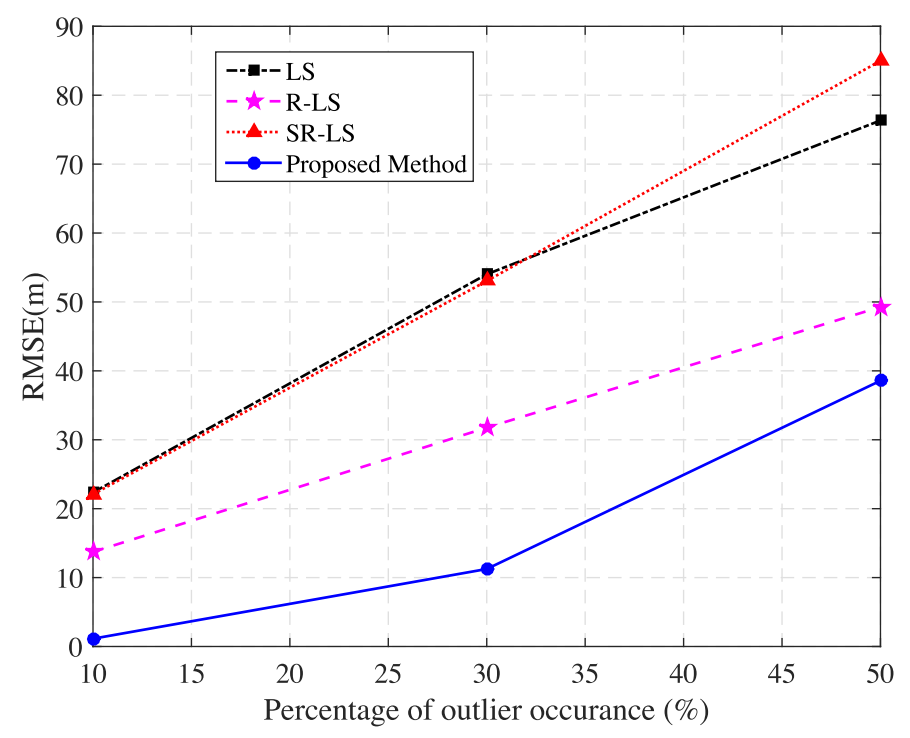

Fig. 2. The RMSE of the robot position estimates through the LS approach of (8), R-LS, SR-LS, and the proposed method with respect to an increasing outliers occurrence rate. The proposed method shows great robustness to the outliers.

and the range measurements considering that $\kappa$ enables the optimization (11) to discard the outliers and find the user's location with the cleaned measurements. The second term renders a sparse estimate of the ranges that contain the outliers. In essence, $\kappa$ absorbs significant deviations from fingerprints. Optimization problem (8) amounts to a second order cone program, which can be efficiently solved. Therefore, (11) jointly estimates the outliers and the agent's position using cleaned range measurements.

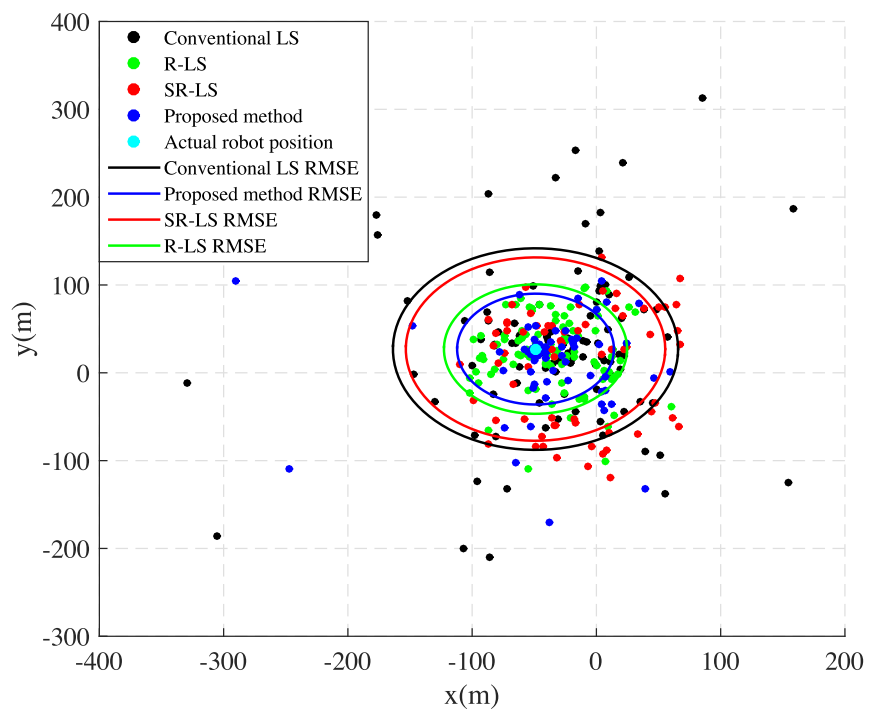

Fig. 3. The distribution of the robot position estimates for LS approach of (8), R-LS, SR-LS, and the proposed method. The robot actual position is the big cyan circle. The estimated poses are mostly concentrated around the actual robot position.

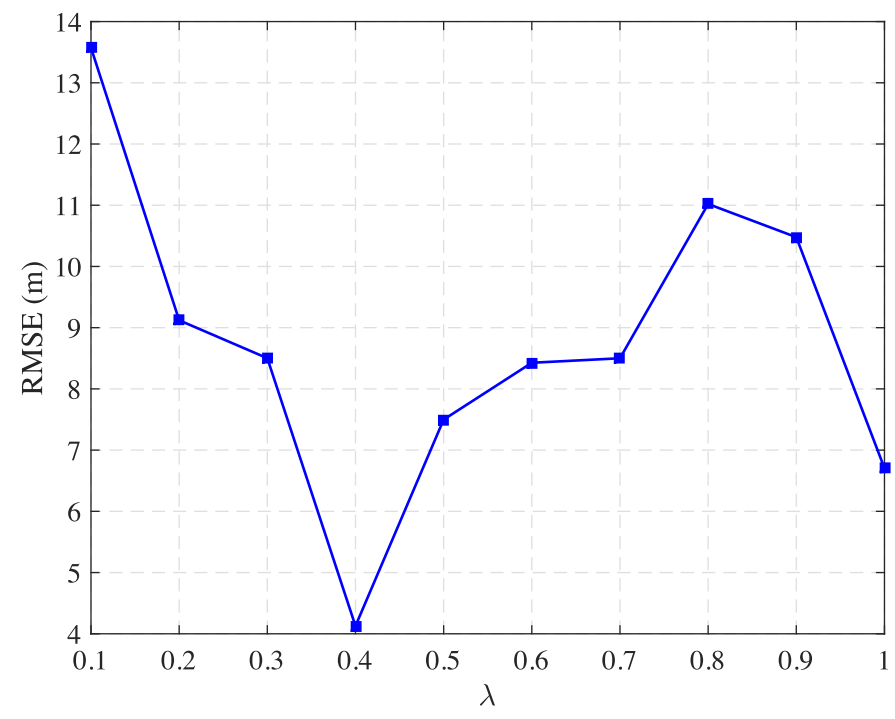

Fig. 4. The performance of the proposed method for different $\lambda$.

\section{RESUlts AND Discussion}

To evaluate the performance of the proposed trilateration method, computer simulations have been conducted through comparing the proposed method with original linearized LS approach [23], R-LS approach, and SR-LS approach [20]. We consider a random anchors geometry where 8 anchors are scattered in $[-50,50] m \times[-50,50] m$ space and the agent is located at $\mathbf{p}=(-49,27)$. The geometry of the anchors and agents is shown in Fig. 1 where the CRLB is 0.46 . The performance of the algorithm has been evaluated through 1000 simulation runs.

The outliers both affect the magnitude and the ranges that contain the outliers. 
The evaluation criteria is based on the expectation over the Euclidean distances between the actual and estimated positions of the agent as

$$
\operatorname{RMSE}=\mathbb{E}\left\{\sqrt{(\hat{\mathbf{p}}-\mathbf{p})^{T}(\hat{\mathbf{p}}-\mathbf{p})}\right\}
$$

known as root mean square error (RMSE), where $\hat{\mathbf{p}}$ is the estimated robot position.

Fig. 2 shows the RMSE of the LS, R-LS, SR-LS, and our proposed method when the percentage of the ranges that contain the outliers increases. The solution of LS and SRLS is highly sensitive if the ranges contain outliers, which makes them impractical in real applications. The R-LS method positioning error increases linearly with the increasing rate of outliers. The least error is for the proposed method where it shows the robustness of the solution to the range outliers. This method works considerably well when the outliers are less than $30 \%$ and has the least error amongst all the compared methods.

For a better illustration, the distribution of the robot position estimates obtained by LS, R-LS, SR-LS, and proposed method is shown when the noise variance is $\sigma^{2}=1$. The actual robot position is also shown with a bigger cyan circle. The concentration of the robot position estimates show that the LS estimates is scattered widely in the area and provides the worst estimates if the ranges contain outliers. The R-LS and SR-LS estimates have quite similar estimates while the blue estimates of the proposed method is mostly concentrated around the true robot location.

The performance of the localization approach of (11) depends on the tuning parameter $\lambda$. Fig. 4 shows the RMSE of the proposed approach for different values of $\lambda$. The optimal is $\lambda=0.4$. The optimal $\lambda$ can be achieved through cross validation $(\mathrm{CV})$ or a training dataset.

We also explored the detection rate of outliers by evaluating single range outliers. Our simulation results show that the outliers have been exactly detected in $\% 97$ of the time.

\section{CONCLUSIONS}

In this paper, we proposed a trialteration method for robot localization which jointly estimates the robot position and defines the ranges that contain the outliers. These outliers are considered as the inordinate errors that occur for different reasons such as system shut down or adversary attacks. The proposed approach reformulates the trilateration problem into an overdetermined set of linear equations. The position estimates is an optimization formulation consisting of a least square component that minimizes the noise effect and an $\ell_{1}$ component that detect the sparse outliers vector. The results show that the proposed approach has a great enhancement over the estimated positions of the robot compared to the existing solutions.

\section{REFERENCES}

[1] M. McGuire, K. Plataniotis, and A. Venetsanopoulos, "Data fusion of power and time measurements for mobile terminal location," IEEE Transactions on Mobile Computing, vol. 4, no. 2, pp. 142-153, March 2005.
[2] P. Misra and P. Enge, Global Positioning System: Signals, Measurements, and Performance, 2nd ed. Ganga-Jamuna Press, Lincoln MA, 2006.

[3] Y. Chon, E. Talipov, and H. Cha, "Autonomous management of everyday places for a personalized location provider," IEEE Transactions on Systems, Man, and Cybernetics, Part C: Applications and Reviews, vol. 42, no. 4, pp. 518-531, July 2012.

[4] Y. Kim, Y. Chon, and H. Cha, "Smartphone-based collaborative and autonomous radio fingerprinting," IEEE Transactions on Systems, Man, and Cybernetics, Part C: Applications and Reviews, vol. 42, no. 1, pp. $112-122$, Jan 2012.

[5] J. Koo and H. Cha, "Unsupervised locating of WiFi access points using smartphones," IEEE Transactions on Systems, Man, and Cybernetics, Part C: Applications and Reviews, vol. 42, no. 6, pp. 1341-1353, Nov 2012.

[6] Y.-T. Chan, W.-Y. Tsui, H.-C. So, and P.-C. Ching, "Time-of-arrival based localization under NLOS conditions," IEEE Transactions on Vehicular Technology, vol. 55, no. 1, pp. 17-24, Jan 2006.

[7] X. Li and K. Pahlavan, "Super-resolution TOA estimation with diversity for indoor geolocation," IEEE Transactions on Wireless Communications, vol. 3, no. 1, pp. 224-234, Jan 2004.

[8] S. He and S. H. G. Chan, "Wi-fi fingerprint-based indoor positioning: Recent advances and comparisons," IEEE Communications Surveys Tutorials, vol. 18, no. 1, pp. 466-490, Firstquarter 2016.

[9] P. Biswas, H. Aghajan, and Y. Ye, "Integration of angle of arrival information for multimodal sensor network localization using semidefinite programming," in In Proceedings of 39th Asilomar Conference on Signals, Systems and Computers, 2005.

[10] D. Niculescu and B. Nath, "Ad hoc positioning system (APS) using AOA," in Twenty-Second Annual Joint Conference of the IEEE Computer and Communications, vol. 3, March 2003, pp. 1734-1743 vol.3.

[11] R. Peng and M. Sichitiu, "Angle of arrival localization for wireless sensor networks," in 3rd Annual IEEE Communications Society on Sensor and Ad Hoc Communications and Networks, vol. 1, Sept 2006, pp. 374-382.

[12] D. Manolakis, "Efficient solution and performance analysis of 3-D position estimation by trilateration," IEEE Transactions on Aerospace and Electronic Systems, vol. 32, no. 4, pp. 1239-1248, Oct 1996.

[13] - "Efficient solution and performance analysis of 3-D position estimation by trilateration," Aerospace and Electronic Systems, IEEE Transactions on, vol. 32, no. 4, pp. 1239-1248, Oct 1996.

[14] W. Murphy and W. Hereman, "Determination of a position in three dimensions using trilateration and approximate distances," Colorado School of Mines, Golden, CO, Tech. Rep., 1995.

[15] B. Denis, J.-B. Pierrot, and C. Abou-Rjeily, "Joint distributed synchronization and positioning in UWB ad hoc networks using TOA," IEEE Transactions on Microwave Theory and Techniques, vol. 54, no. 4, pp. 1896-1911, June 2006.

[16] Y. Shen and M. Win, "Fundamental limits of wideband localization-part I: A general framework," IEEE Transactions on Information Theory, vol. 56, no. 10, pp. 4956-4980, Oct 2010.

[17] Y. Shen, H. Wymeersch, and M. Win, "Fundamental limits of wideband localization-part II: Cooperative networks," IEEE Transactions on Information Theory, vol. 56, no. 10, pp. 4981-5000, Oct 2010.

[18] H. V. Trees, Detection, Estimation, and Modulation Theory, Part I. New York: Wiley, 1968.

[19] T. Van Nguyen, Y. Jeong, H. Shin, and M. Win, "Least square cooperative localization," IEEE Transactions on Vehicular Technology, vol. 64, no. 4, pp. 1318-1330, April 2015.

[20] A. Beck, P. Stoica, and J. Li, "Exact and approximate solutions of source localization problems," IEEE Transactions on Signal Processing, vol. 56, no. 5, pp. 1770-1778, May 2008.

[21] E. Larsson and D. Danev, "Accuracy comparison of LS and squaredrange LS for source localization," IEEE Transactions on Signal Processing, vol. 58, no. 2, pp. 916-923, Feb 2010.

[22] K. Cheung, W.-K. Ma, and H. So, "Accurate approximation algorithm for TOA-based maximum likelihood mobile location using semidefinite programming," in IEEE International Conference on Acoustics, Speech, and Signal Processing, vol. 2, May 2004, pp. ii-145-8 vol.2.

[23] K. Cheung, H. So, W.-K. Ma, and Y. Chan, "Least squares algorithms for time-of-arrival-based mobile location," IEEE Transactions on Signal Processing, vol. 52, no. 4, pp. 1121-1130, April 2004. 